\title{
DISCUSSION
}

\section{Stability of intact slopes with tensile strength cut-off}

\author{
RADOSLAW L. MICHALOWSKI* and STEFANO UTILI†
}

\section{Contribution by Stefano Utili}

The discusser would like to raise three main points of discussion. These are taken in turn below.

The first point is: are limit analysis (LA) solutions (drained case) based on non-physical values of dilation angle of any use?

In the abstract the author argues that 'Tension cut-off used in the analysis permits deformation modes with the dilatancy angle exceeding the internal friction angle' (Michalowski, 2017), but actually the tension cut-off used in the analysis requires deformation modes with the dilatancy angle exceeding the internal friction angle, $\phi$, more commonly called the angle of shearing resistance in the literature; in the opinion of the discusser, this is a great weakness of the analysis presented because it is well documented that geomaterials exhibit dilation angles smaller than the angle of shearing resistance (e.g. Bolton, 1986; Powrie, 2004). The values of dilation angle assumed in the analysis are up to $90^{\circ}$, which is almost an order of magnitude larger than the values measured in experiments for most soils. This assumption on the dilation angle leads in turn to unrealistic failure mechanisms, which feature an overhanging profile for the new slope profile left after the occurrence of the failure mechanism (see Figs 3(a) and 7(a)). It may be argued that according to the upper bound theorem, failure mechanisms need only to be kinematically admissible and their geometry does not need to reflect the geometry of the true failure mechanism; however, here the issue is that the least upper bound is found among a class of mechanisms that are considered kinematically compatible only because an unrealistic assumption on a key material property, dilation, is made, because if a realistic assumption on such a material property were made, these mechanisms would not appear among the compatible ones.

The second point concerns inconsistencies in some key assumptions made in the analysis presented. In the analysis, dilation is assumed to vary in the tensile zone of the slope according to an equation postulated by the author (see equation (8)), whereas it is assumed uniform and equal to the angle of shearing resistance in the compressive zone of the slope. Yet the angle of shearing resistance and cohesion are assumed uniform throughout the whole slope. According to basic soil mechanics, the angle of shearing resistance is not independent of dilation but instead a strong correlation between the two exists (see for instance Bolton (1986)). Therefore, the assumption of a geomaterial that in the compressive zone of the slope exhibits a dilation angle equal to the angle of shearing resistance, which implies a precise relation between dilation and friction, and instead in another part of the slope (upper tensile part) exhibits a spatially varying dilation angle and a uniform angle of shearing

\footnotetext{
* Department of Civil and Environmental Engineering, University of Michigan, Ann Arbor, MI, USA (Orcid:0000-0002-9557-4802). $\uparrow$ School of Engineering, Newcastle University, Newcastle upon Tyne, UK.
}

resistance, which implies that dilation and shearing resistance are completely uncorrelated, is utterly inconsistent and goes against experimental evidence.

Also in the opinion of the discusser another inconsistency resides in the fact that the values of the angle of dilation in the tensile zone of the slope are found as part of the solution in the search for the minimum slope stability factor, whereas the angle of dilation in the lower part of the slope and the angle of shearing resistance in the whole slope are assumed as known material properties. This choice seems arbitrary.

The third point is: are LA solutions including a rupture mode (undrained case) rigorous bounds? In the undrained analyses presented, the author argues that the volumetric deformation required for the validity of the normality rule in the upper part of the slope (Fig. 7) 'can be understood as a rupture in a saturated soil' so that the soil can still be considered as incompressible and consistency with the undrained nature of the analysis to be preserved. The discusser notes that a 'rupture mode' taking place as part of the slope failure mechanism implies that the continuity of the medium considered in the energy balance equation is violated. Since continuity is a key requirement for the applicability of the limit theorems (Drucker \& Prager, 1952; Drucker et al., 1952), lack of continuity implies the theorems are no longer applicable and therefore the solutions presented cannot be considered upper bounds to the limit load. For the sake of completeness, non-applicability of the limit theorems also concerns cracks forming as part of the failure mechanism (Michalowski, 2013) but does not apply to cracks already present in the slope (Utili \& Abd, 2016; Abd \& Utili, 2017). These are also called pre-existing cracks as opposed to cracks forming as part of the failure mechanism, since pre-existing cracks define the geometry of the slope - that is, the medium considered, rather than being generated as part of the failure mechanism. Thus the discusser argues that any solution including energy dissipated by the formation of a rupture band as part of the failure mechanism is not rigorous, owing to violation of the continuity condition, whereas in the case where the crack or rupture band is part of the initial geometry of the medium considered, and so pre-existing the formation of the limit analysis kinematic mechanism, the solution provides a rigorous bound.

\section{Author's reply}

The author appreciates the interest of the discusser in the subject, but does not agree with the statements in the three points raised in the written discussion.

Point 1. The first comment is somewhat broad, and it relates to the limit analysis solutions that imply large volumetric deformation, referred to by Utili as 'non-physical' dilation. Although the published note relates to geomaterials with tensile strength or tensile strength cut-off (bonded materials), it is useful to look first at the dilatancy of granular media, with an early description provided by Reynolds (1885). This 'change of bulk' (Reynolds, 1885) was quantified later in terms of dilatancy angle $\psi$ (e.g. Hansen, 1958), which 
for plane-strain deformation processes can be defined as $\left(\dot{\varepsilon}_{1}, \dot{\varepsilon}_{3}\right.$ are the in-plane principal strain rates)

$$
\sin \psi=-\frac{\dot{\varepsilon}_{1}+\dot{\varepsilon}_{3}}{\dot{\varepsilon}_{1}-\dot{\varepsilon}_{3}}
$$

Proving the theorems of limit analysis requires the postulate of maximum plastic work, which implies that the material is perfectly plastic, and is governed by a convex limit state condition and the normality flow rule. Sand is a pressure-dependent (frictional) geomaterial and, if described as an associative perfectly plastic material, equation (21) will yield the dilatancy angle equal to the angle of shearing resistance. However, the true dilation angle accounts for only a portion of the shearing resistance angle. Once the limit state condition and the plastic flow rule are defined, the angle of dilation is no longer an independent parameter. Consequently, the associative model used in limit analysis overestimates dilatancy for granular materials (with the exception of undrained failure processes where deformation is incompressible, $\psi=0$ ). Associativity also overestimates dilatancy of bonded geomaterials. Many textbook solutions to stability problems involving a drained deformation process (e.g. Chen, 1975; Atkinson, 2007) have been used in engineering practice, despite this 'non-physical' dilatancy predicted by the model involved in the analysis. When seeking active limit loads, the kinematic approach yields an upper bound to the true limit load. If the deformation of the material is governed by the non-associative flow rule, the solutions based on mechanisms conforming to the normality flow rule still provide rigorous upper bounds (Radenkovic, 1962).

It might be of interest to note that solutions for non-associative materials can be estimated by defining a 'fictitious material' which accounts for non-associativity (Drescher \& Detournay, 1993). The method was developed for rigid-block mechanisms, in which the solutions following from the work rate balance equation and those from equilibrium of forces can be proved identical (Michalowski, 1989). As expected, this method indicates that the kinematic solutions to active load for non-associative materials are lower than those for associative ones, but there is no proof that these solutions are rigorous bounds to the true solutions.

Bonded geomaterials have the ability to resist tension, but tensile strength is not a matter of routine testing. Introducing a separate tensile strength limitation from the limit on the shear strength was a reasonable step in describing strength for bonded geomaterials or weathered rocks (Drucker \& Prager, 1952), and the note discussed here presented an example of how this limitation can be included in a stability analysis. The plastic flow rule associated with the non-linear section of the failure criterion (tension cut-off) for bonded materials indicates that failure modes with large volumetric strain components can be modelled. These are not necessarily 'non-physical'. Such modes have been observed in porous solids, both in sand and in bonded materials; they are a form of localised strain, referred to as 'dilation bands' (Du Bernard et al., 2002; Aydin et al., 2006; Deniz Akin \& Likos, 2017). Much of the argument by the discusser relates to sand, whereas the published note was about intact slopes in material with tensile strength or tensile strength cut-off (bonded materials). Some of the confusion may have been caused by using the term 'dilation angle' in regard to angle $\delta$ in Fig. 2(a). In more recent papers (e.g. Park \& Michalowski, 2017), this angle was referred to as a 'rupture angle' (rather than a dilation angle), to emphasise that this mode of plastic deformation is distinct from Reynolds' dilatancy or the change in the density associated with grain interlocking in sand referred to by Taylor (1948) in the context of shearing of granular media.

At the end of his first comment, Utili refers to 'kinematically compatible' mechanisms, presumably, kinematically admissible mechanisms. One of the requirements of kinematical admissibility is consistency (or compatibility) of the velocity field with the normality flow rule. Once the strength envelope was defined as illustrated in Fig. 2 of the original note, the mechanism used in the analysis, Fig. 3, is strictly admissible, and the analysis yields a rigorous bound to the stability factor. The discusser makes a comment that a large dilatancy leads 'to unrealistic failure mechanisms'. Limit analysis can yield rigorous bounds to limit loads, but the mechanism might not necessarily resemble a true failure mechanism. The expectation that it should is reasonable, but it is an intuitive conjecture. Attainment of the best solution is based on the optimisation process where the mechanism geometry is varied, and not on the judgement of how reasonable the mechanism seems to be. The discusser views the mechanisms illustrated in Figs 3(a) and 7(a) as unrealistic. This follows from misinterpretation of kinematic solutions of limit analysis in trying to argue that the solutions presented would be unrealistic in leaving the slope with an 'overhanging profile'. Limit analysis can only consider an incipient failure. Once instability takes place, the failure process of slopes evolves and typically ends with debris flow; a failure process resembling toppling may occur in the portion governed by the tension cut-off. Consequently, the profile left over is very different from the shape of the mechanism that started the collapse. However, assessment of geometry of boundaries can be attempted using kinematic limit analysis in steady-state or self-similar problems (a class of problems typical in plastic forming of metals, but also applicable to bulk materials (Drescher \& Michalowski, 1984)). In any case, one should not expect a failure mechanism to exactly resemble the true collapse pattern, because limit analysis is based on a simplified (associative and perfectly plastic) material model.

To address a rather general opening question in the first comment, it might be useful to bring the classical PrandtlReissner solution to the limit load on a pressure-dependent medium (Prandtl, 1920; Reissner, 1924). Although the solution was originally obtained using the slip-line method, an identical solution can be obtained using the kinematic approach of limit analysis. Despite a significant overestimation of the dilatancy of soils by the normality flow rule, this solution (amended by a term dependent on the soil weight) is part of many standards for calculating bearing capacity of footings. In conclusion, whether the true dilation angle is overestimated by the normality flow rule in plastic shearing of soils or the large volumetric strain rates are considered in rupture modes, limit analysis solutions appear to be useful. The reader will find more discussion supporting this conclusion in the response to the third comment.

Point 2. In his second comment, Utili disputes some of the details of the solution presented in the note. The discusser makes a series of imprecise statements - for example, the 'dilatancy is assumed to vary' in some portion of the slope, whereas it is 'assumed uniform' elsewhere. None of these distributions was assumed. The material in the entire slope is uniform, but with a non-linear strength envelope; the material's behaviour is governed here by material functions, not constants. Consequently, the material can exhibit different behaviour in different portions of the slope, depending on the stress state. None of the behaviour was pre-determined (assumed), but the material was allowed to choose the mode of deformation, which assured the best (least) solution. Some solutions were found for cases where the entire slope was failing with a uniform angle of shearing resistance, in some cases the entire slope was subjected to rupture mode 
(with varying rupture angle $\delta$ ), whereas in many cases the best solution required a combination of the two. Regarding equation (8): the specific values of angle $\delta$ were not assumed, only a linearity of its distribution with angular co-ordinate $\theta$ was postulated for convenience. This was done in lieu of explicitly varying the geometry of the failure surface, which was found to be more elaborate. Non-linear distributions postulated in the analysis provided indistinguishable solutions.

The remaining part of the second comment is a result of confusion regarding what limit analysis is, what its theoretical underpinnings are and what its limitations are. In contrast to finite-element analysis, where a more elaborate material model can be used, limit analysis is based on perfect plasticity with a convex failure criterion and normality plastic flow rule. Geomaterials are neither perfectly plastic nor associative, thus some discrepancy between the true behaviour and the behaviour of the model used in limit analysis is inevitable. True dilatancy of geomaterials and that following from normality of plastic flow cannot be reconciled. There are other limitations in the method, in particular, the inability to address progressive failure. However, the kinematic theorem can be used to find an upper bound to an active load causing failure of a structure (or stability factor as defined in the note). Even if the true material is not governed by the associative flow rule, the solution can still be proven to be an upper bound (Radenkovic, 1962). Hence, there is value in such solutions.

The discusser made a comment that the dilatancy angle and the angle of shearing resistance are not correlated in the solution, which is against experimental evidence. A strict limit analysis can be performed only for a material governed by the associative plastic flow rule, and the dilatancy in such models follows uniquely from that rule. The true dilatancy is typically much smaller, and this is the case for models with or without tensile strength cut-off alike. Limit analysis is an approximate method, yielding bounds to the true solution, and it is based on the normality rule of plastic flow.

In a long sentence at the end of the second comment, Utili makes a bizarre (and somewhat repetitive) remark, seeing an inconsistency in the angles of dilation and shearing resistance being 'assumed as known material properties' in the lower portion of the slope, but being a part of the solution in the upper portion. In order to solve any mechanics boundary value problem, material properties have to be known. Material properties in the entire slope were known and they were uniform. The discusser seems to consider geomaterials as media governed by material constants. However, the strength envelope of the material in the note is non-linear; the behaviour is governed by material functions, and, depending of the stress state, the material can exhibit different deformation modes at any point in the slope as long as they do not violate admissibility of the failure mechanism. Finding the specific values of the material functions in the deformation field is an actual challenge. This is why in many papers where the Hoek-Brown strength criterion is used, the non-linear strength envelope is first replaced by a linear approximation (circumscribing the non-linear failure criterion), and the solutions are found using a material described by constants rather than material functions. An early suggestion of that kind was published by Drescher \& Christopoulos (1988), followed by many others (e.g. Yang \& Yin, 2004). The note published takes advantage of non-linearity of the strength envelope to find a better solution than those based on a linear approximation.

Point 3. In his last point, the discusser argues that limit analysis cannot be used when rupture modes are part of the failure mechanism. This is an odd statement. First, Utili cites the paper of Drucker \& Prager (1952) to support his statement, but it was in this very paper where Drucker and
Prager introduced a tension cut-off and used rupture modes associated with it in limit analysis of a slope. Second, in recent papers of the discusser (Abd \& Utili, 2017; Voulgari \& Utili, 2017), the very same model was adopted, rupture modes were considered in limit analysis and a procedure was used that Utili seems now to denounce.

Kinematic discontinuities are interpreted in limit analysis as bands with large velocity gradients (Drucker et al., 1952) and are idealised in computations as zero-thickness layers. The layers of localised shear strain are typically referred to as shear bands, whereas the term 'dilation bands' was coined more recently for the layers of localised volumetric increase (Du Bernard et al., 2002; Aydin et al., 2006). The latter are distinct from fractures (cracks) considered in linear elastic fracture mechanics. The appearance of a similar band was detected in a Brazilian test on an oven-dried clay prior to the appearance of a tensile crack (Deniz Akin \& Likos, 2017). Contrary to the statement by the discusser, the kinematic limit analysis is applicable to both types of localised deformation. As to the rupture modes in undrained processes, they are conceivable, but, so far, hypothetical.

The note related to intact slopes, but Utili broadened the discussion to slopes with cracks and their possible formation as part of the collapse mechanism. This subject has been discussed elsewhere (Michalowski, 2014), and it was remarked unequivocally that 'propagation of a crack cannot be modelled using limit analysis' and the subject might be better understood if the term 'crack opening' was replaced with the concept of a 'band' deformation. As argued above, limit analysis can be applied to mechanisms with localised deformation. Situations where the analysis cannot be applied are in problems with existing cracks of unknown location, as those considered in Utili (2013). Such problems are ill-posed, but that issue was also discussed earlier (Michalowski, 2014).

One of the fundamental assumptions in limit analysis is perfect plasticity (implying ductility of deformation), whereas bonded geomaterials under low confining stresses exhibit some brittleness, similar to concrete. Chen (1975: pp. 451-452) argued that if the tensile strength of rock or concrete is assumed to be zero, then 'the limit theorems of perfect plasticity will hold rigorously for this idealisation. They can be applied easily when tensile crack is introduced in the failure mechanism.' He further comments that 'The assumption of infinite ductility in tension at zero strength is rigorous and conservative for the application of limit analysis...' (Chen, 1975: p. 452). Chen also remarks that the assumption of ductility in compression is more questionable, but if the strain of the material (concrete/rock) is small prior to a brittle drop in stress then the deformability 'may be sufficient to permit the consideration of limit theorems' (Chen, 1975: p. 452). Consequently, examples can be found in the literature where limit analysis has been applied to materials such as rocks and concrete (e.g. Chen \& Drucker, 1969; Michalowski, 1985; Nielsen, 1999).

Finally, it is worthwhile commenting on a special type of discontinuous mechanism of deformation where the material separates from the boundary. Such deformation modes have been used with success in limit analysis of problems with frictional boundaries. Collins (1969: p. 323) argued that the definition of kinematical admissibility of the velocity fields is 'unnecessarily restrictive for the validity of the upper bound inequality of limit analysis'. The specific examples considered by Collins were compression and extrusion of metals. Typically, the rate of work dissipation at frictional boundaries cannot be calculated directly, but when requiring the velocities to be inclined to the boundaries at the angle of friction, the dissipation on sliding along boundaries becomes exactly nil, and the solution to the unknown limit load can be found. While the velocity indicating separation from the 
boundary is not realistic, the procedure does not violate any of the limit analysis prerequisites, and the outcome is a strict bound to the limit load. The same method was used independently by Mróz \& Drescher (1969) in calculations of loads on frictional walls of bins and hoppers produced by bulk materials. Collins (1973) also applied this technique to calculate the load on a rough retaining wall. In all cases the material in the considered mechanism indicates separation from the boundary, but a rigorous bound was obtained. Even though the frictional sliding rule is non-associative, the use of the normality rule (Michalowski \& Mróz, 1978) is consistent with limit analysis and it leads to useful solutions.

\section{REFERENCES}

Abd, A. \& Utili, S. (2017). Design of geosynthetic - reinforced slopes in cohesive backfills. Geotextiles and Geomembranes 45, $627-641$.

Atkinson, J. (2007). The mechanics of soils and foundations, 2nd edn. London, UK: Taylor \& Francis

Aydin, A., Borja, R. I. \& Eichhub, P. (2006). Geological and mathematical framework for failure modes in granular rock. J. Struct. Geol. 28, No. 1, 83-98.

Bolton, M. D. (1986). The strength and dilatancy of sand. Géotechnique 36, No. 1, 65-78, https://doi.org/10.1680/ geot.1986.36.1.65.

Chen, W. F. (1975). Limit analysis and soil plasticity. Amsterdam, the Netherlands: Elsevier.

Chen, W. F. \& Drucker, D. C. (1969). Bearing capacity of concrete blocks or rock. J. Engng Mech. Div. 95, No. 4, 955-978.

Collins, I. F. (1969). The upper bound theorem for rigid/plastic solids generalized to include Coulomb friction. J. Mech. Phys. Solids 17, No. 5, 323-338.

Collins, I. F. (1973). A note on the interpretation of Coulomb's analysis of the thrust on a rough retaining wall in terms of the limit theorems of plasticity theory. Géotechnique 23, No. 3, 442-447, https://doi.org/10.1680/geot.1973.23.3.442.

Deniz Akin, I. \& Likos, W. J. (2017). Brazilian tensile strength testing of compacted clay. Geotech. Testing J. 40, No. 4, 608-617.

Drescher, A. \& Christopoulos, C. (1988). Limit analysis slope stability with nonlinear yield condition. J. Numer. Analyt. Methods Geomech. 12, No. 3, 341-345.

Drescher, A. \& Detournay, E. (1993). Limit load in translational failure mechanisms for associative and non-associative materials. Géotechnique 43, No. 3, 443-456, https://doi.org/ 10.1680/geot.1993.43.3.443.

Drescher, A. \& Michalowski, R. L. (1984). Density variation in pseudo-steady plastic flow of granular media. Géotechnique 34, No. 1, 1-10, https://doi.org/10.1680/geot.1984.34.1.1.

Drucker, D. C. \& Prager, W. (1952). Soil mechanics and plastic analysis for limit design. Q. Appl. Math. 10, No. 2, 157-165.

Drucker, D. C., Prager, W. \& Greenberg, H. J. (1952). Extended limit design theorems for continuous media. Q. Appl. Math. 9, No. 4, 381-389.

Du Bernard, X., Eichhub, P. \& Aydin, A. (2002). Dilation bands: a new form of localized failure in granular media. Geophys. Res. Lett. 29, No. 24, 2176, https://doi.org/10.1029/2002GL015966.

Hansen, B. (1958). Line ruptures regarded as narrow rupture zones. Basic equations based on kinematic considerations. Proceedings of Brussels conference 58 on earth pressure problems, Brussels, Belgium, vol. 1, pp. 39-48.

Michalowski, R. L. (1985). Limit analysis of quasi-static pyramidal indentation of rock. Int. J. Rock Mech. Min. Sci. 22, No. 1, $31-38$.

Michalowski, R. L. (1989). Three-dimensional analysis of locally loaded slopes. Géotechnique 39, No. 1, 27-38, https://doi.org/ 10.1680/geot.1989.39.1.27.

Michalowski, R. L. (2013). Stability assessment of slopes with cracks using limit analysis. Can. Geotech. J. 50, No. 10, 1011-1021, https://doi.org/10.1139/cgj-2012-0448.

Michalowski, R. L. (2014). Reply to the discussion by Utili on 'Stability assessment of slopes with cracks using limit analysis'. Can. Geotech. J. 51, No. 7, 826-827.

Michalowski, R. L. (2017). Stability of intact slopes with tensile strength cut-off. Géotechnique 67, No. 8, 720-727, https:// doi.org/10.1680/jgeot.16.P.037.

Michalowski, R. L. \& Mróz, Z. (1978). Associated and nonassociated sliding rules in contact friction problems. Arch. Mech. Stos. (Archives of Mechanics) 30, No. 3, 259-276.

Mróz,. Z. \& Drescher, A. (1969). Limit plasticity approach to some cases of flow of bulk solids. J. Engng Ind. 91, No. 2, 357-364.

Nielsen, M. P. (1999). Limit analysis and concrete plasticity, 2nd edn. New York, NY, USA: CRC Press.

Park, D. \& Michalowski, R. L. (2017). Three-dimensional stability analysis of slopes in hard soil/soft rock with tensile strength cut-off. Engng Geol. 229, 73-84.

Powrie, W. (2004). Soil mechanics: concepts and applications, 2nd edn. Abingdon, UK: Spon Press.

Prandtl, L. (1920). Über die Härte plastischer Körper. Nachrichten von der Gesellschaft der Wissenschaften zu Göttingen, Mathematische-Physikalische Klasse 1920, 74-85 (in German).

Radenkovic, D. (1962). Théorie des charges limites extension a la mécanique des sols. In Séminaire de plasticité (École Polytechnique, 1961) (ed. J. Mandel), Publications scientifiques et techniques du Ministère de l'Air/Notes techniques, no. 116, pp. 129-141. Paris, France: Service de Documentation et d'Information Technique de l'Aéronautique (in French).

Reissner, H. (1924). Zum Erddruckproblem. In Proceedings of the 1st international congress for applied mechanics (eds C. B. Biezeno and J. M. Burgers), pp. 295-311. Delft, the Netherlands: Technische Boekhandel en Drukkerij, J. Waltman $\mathrm{Jr}$ (in German).

Reynolds, O. (1885). On the dilatancy of media composed of rigid particles in contact. With experimental illustrations. Phil. Mag. 20, No. 127, 469-482.

Taylor, D. W. (1948). Fundamentals of soil mechanics. New York, NY, USA: Wiley.

Utili, S. (2013). Investigation by limit analysis on the stability of slopes with cracks. Géotechnique 63, No. 2, 140-154, https://doi.org/10.1680/geot.11.P.068.

Utili, S. \& Abd, A. H. (2016). On the stability of fissured slopes subject to seismic action. Int. J. Numer. Analyt. Methods Geomech. 40, No. 5, 785-806.

Voulgari, C. \& Utili, S. (2017). A general analytical solution for the evolution of cliffs accounting for strength degradation, seismic action, formation of tension cracks and seepage. Engng Geol. 219, 92-106.

Yang, X. L. \& Yin, J. H. (2004). Slope stability analysis with nonlinear failure criterion. J. Engng Mech. 130, No. 3, 267-273. 